2. To: (Receiving Organization)

\section{DISTRIBUTION}

5. Proj./Prog./Dept./Div.:

HANDI 2000

8. Originator Remarks:

KEY WORDS: H2K, BMS, HANDI 2000, PEOPLESOFT GENERAL LEDGER, PROJECT COSTING, BUSINESS SYSTEM IMPLEMENTATION, FINANCE, AS-IS/TO-BE

11. Receiver Remarks:

3. From: (Originating Organization)
SYSTEMS FLUOR DANIEI HANFORD
6. Design Authority/Design AgentCOg. Engr.:
DAWN E. ADAMS

DAWN E. ADAMS

4. Related EDT No:

N/A

7. Purchase Order No.:

N/A

9. Equip./Component No.:

$\mathrm{N} / \mathrm{A}$

10. System/Bidg./Facility:

$\mathrm{N} / \mathrm{A}$

12. Major Assm. Dwg. No.:

$\mathrm{N} / \mathrm{A}$

13. Permit/Permit Application No.:

N $/ \mathrm{A}$

14. Required Response Date:

N $/ A$

\begin{tabular}{|c|c|c|c|c|c|c|c|c|c|c|c|c|c|c|}
\hline & & & & & & & & & & & & & & \\
\hline 15. & & & & & DATA TRE & ANSMITTE & & & & & (F) & (G) & $(\mathrm{H})$ & (1) \\
\hline $\begin{array}{l}\text { (A) } \\
\text { hem } \\
\text { No. }\end{array}$ & & (B) Docume & ent/Drawing & & $\begin{array}{l}\text { (C) Sheet } \\
\text { No. }\end{array}$ & $\begin{array}{l}\text { (D) Rev. } \\
\text { No. }\end{array}$ & (E) $\mathrm{T}$ & Title or D & Descripti & ion of Data Transmitted & $\begin{array}{l}\text { Approval } \\
\text { Desig- } \\
\text { nator }\end{array}$ & $\begin{array}{c}\text { Reason } \\
\text { for Trans- } \\
\text { mittal }\end{array}$ & $\begin{array}{c}\text { Origi- } \\
\text { nator } \\
\text { Dispo- } \\
\text { stion }\end{array}$ & \begin{tabular}{|l} 
Receiv- \\
er \\
Dispo- \\
sition
\end{tabular} \\
\hline 1 & HNE - & -2640 & & & N/A & 0 & ACC & SRUALS & $S$ FOR & HANDI 2000 & & & & \\
\hline & & & & & & & BUS & INESS & $S$ MAN & TAGEMENT SYSTEM & $N / A$ & 2 & & \\
\hline 2 & HNF- & -2641 & & & $\mathrm{~N} / \mathrm{A}$ & 0 & ALLS & OCATI & IONS & FOR HANDI 2000 & & & & \\
\hline & & $\cdot$ & & & & - & BUS: & INESS & $S$ MAN & TAGEMENT SYSTEM & $\mathrm{N} / \mathrm{A}$ & 2 & & \\
\hline 3 & HNE- & -2642 & & & $\mathrm{~N} / \mathrm{A}$ & 0 & BUDS & GET F & FOR $\mathrm{H}$ & IANDI 2000 & & . & & \\
\hline & & & & & & & BUS & INESS & $S$ MAN & IAGEMENT SYSTEM & $\mathrm{N} / \mathrm{A}$ & 2 & & \\
\hline & (SEE & SHEET & 2) & & & & & & & & & & & \\
\hline 16. & & & & & & & & KEY & & & & & & \\
\hline Appro & val Des & ignator $(\boldsymbol{F})$ & & & ason for Tran & nsmittal (G & & & & & isposition & (H) \& (I) & & \\
\hline Ese & $\begin{array}{l}\text { S, Q, D } \\
\text { WHC. } \\
\text { Sec. } 1\end{array}$ & $\begin{array}{l}\text { OR N/A } \\
2.7-3-5\end{array}$ & $\begin{array}{l}\text { 1. Appro } \\
\text { 2. Releas } \\
\text { 3. Inform }\end{array}$ & $\begin{array}{l}\text { val } \\
\text { se } \\
\text { ation }\end{array}$ & $\begin{array}{l}\text { 4. Review } \\
\text { 5. Post-Re } \\
\text { 6. Dist. (Re }\end{array}$ & $\begin{array}{l}\text { view } \\
\text { vecipt Ack }\end{array}$ & now. $R$ & Required & & $\begin{array}{l}\text { 1. Approved } \\
\text { 2. Approved w/commer } \\
\text { 3. Disapproved w/comr }\end{array}$ & ent & $\begin{array}{l}\text { 4. Review } \\
\text { 5. Review } \\
\text { 6. Receip }\end{array}$ & $\begin{array}{l}\text { red no/com } \\
\text { ed w/com } \\
\text { t acknowle }\end{array}$ & $\begin{array}{l}\text { mment } \\
\text { iment } \\
\text { ledged }\end{array}$ \\
\hline 17. & & & & & & $\begin{array}{r}\text { SIG } \\
\text { e Approva }\end{array}$ & $\begin{array}{l}\text { SNATUF } \\
\text { a Desig }\end{array}$ & $\begin{array}{l}\text { REIDIST } \\
\text { gnator fo }\end{array}$ & $\begin{array}{l}\text { TRIBUT } \\
\text { or requir }\end{array}$ & ION & 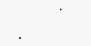 & & & \\
\hline $\begin{array}{l}\text { (G) } \\
\text { Rea- } \\
\text { Son }\end{array}$ & Disp. & (J) Name & $\infty$ & (K) Sig & ire (L) $\mathrm{D}$ & late (M) & $M S I N$ & \begin{tabular}{|c|} 
(G) \\
Rea- \\
son \\
\end{tabular} & $\begin{array}{l}\text { (H) } \\
\text { Disp. }\end{array}$ & (J) Name & (K) Signat & ture & _) Date & (M) MSIN \\
\hline 2 & 1 & Design AU & thority $(1)$ & and & donts & 81998 & $6-21$ & & & Central Fil & $(2)$ & $B /-O$ & & \\
\hline & & Design $\mathrm{Ag}$ & & & & & & & & - & & & & \\
\hline & & Cog. Eng. & & & & & & & & & & & & \\
\hline & & Cog. Mgr. & & & & & & & & & & & & \\
\hline & & QA & & & & & & & & & & & & \\
\hline & & Safety & & & & & & & & & & & & \\
\hline & & Env. & & & & & & & & & & . & & \\
\hline A1) & inel & EDT Lese & $\begin{array}{l}\text { G1-21 } \\
\frac{8-12-98}{\text { Date }}\end{array}$ & $\begin{array}{l}19 . \\
\frac{N / A}{A u t h} \\
\text { for } R\end{array}$ & $\begin{array}{l}\text { edRepresen } \\
\text { iving Organiz }\end{array}$ & $\begin{array}{l}\text { tative } \\
\text { zation }\end{array}$ & Date & $\frac{\text { Uaj }}{\text { Desig }}$ & $\frac{1}{\text { gn Authe }}$ & 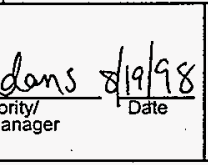 & $\begin{array}{r}\text { 21. DOE } \\
\text { Ctr } \\
\square \\
\square \\
\square\end{array}$ & $\begin{array}{l}\text { EAPPROV } \\
\text { No. } \\
\text { Approved } \\
\text { Approved } \\
\text { Disapprov }\end{array}$ & $\begin{array}{l}\text { w/commer } \\
\text { ed w/comr }\end{array}$ & imts \\
\hline
\end{tabular}




\section{ENGINEERING DATA TRANSMITTAL}

(CONTINUATION PAGE)

\section{Proj./Prog./Dept.Div.:}

HANDI 2000
6. Cog. Eng.

DAWN E. ADAMS
1. EDT

625358

15.

(A)

Item
No.

4

$4 \quad \mathrm{HNF}-2746$

HNF -2643

(1)

$6 \quad$ HNF -2644

\begin{tabular}{c|c|} 
& \\
\hline & \\
\hline 7
\end{tabular}

$7 \quad$ HNF -2645

\begin{tabular}{l|l}
\hline & \\
\hline 8 & HNF -2645 \\
\hline
\end{tabular}

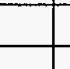

9

HNF -2647

11 HNF -2649

12 HNF -2650

13 HNF -2651

\section{DATA TRANSMITTED}

\begin{tabular}{|c|c|}
\hline $\begin{array}{c}\text { (C) } \\
\text { Sheet } \\
\text { No. }\end{array}$ & $\begin{array}{c}\text { (D) } \\
\text { Rev. } \\
\text { No. }\end{array}$ \\
\hline N/A & 0 \\
\hline & \\
\hline &
\end{tabular}

FIT GAP ANALYSIS FOR

HANDI 2000 BUSINESS

MANAGEMENT SYSTEM

CLOSING PROCEDURE FOR

HANDI 2000 BUSINESS

MANAGEMENT SYSTEM

FIXED ASSETS ACCOUNTING

FOR HANDI 2000 BUSINESS

MANAGEMENT SYSTEM

FUNDS CONTROL FOR HANDI

2000 BUSINESS MANAGEMENT

SYSTEM

GL CHART OF ACCOUNTS

MAINTENANCE FOR HANDT

2000 BUSINESS MANAGEMENT

SYSTEM

HANFORD BUSINESS

STRUCTURE FOR BUSINESS

MANAGEMENT SYSTEM

INVENTORY PROCESSES

FOR HANDI 2000 BUSINESS

MANAGEMENT SYSTEM

INVOICE/DISCAS FOR HANDI

2000 BUSINESS MANAGEMENT

SYSTEM

LABOR COSTING FOR HANDI

2000 BUSINESS MANAGEMENT

SYSTEM

PASSPORT-PEOPLESOFT

INTEGRATION FOR HANDI

2000 BUSINESS MANAGEMENT

SYSTEM

\begin{tabular}{l|l}
$\mathrm{N} / \mathrm{A}$ & 0 \\
\hline
\end{tabular}

Page 2 of 4

\begin{tabular}{|c|c|c|c|}
\hline$(F)$ & $(G)$ & $(H)$ & $(I)$ \\
\hline $\begin{array}{c}\text { Approval } \\
\text { Desig- } \\
\text { nator }\end{array}$ & $\begin{array}{c}\text { Reason } \\
\text { for } \\
\text { Trans- } \\
\text { mittal }\end{array}$ & $\begin{array}{c}\text { Origi- } \\
\text { nator } \\
\text { Dispo- } \\
\text { stion }\end{array}$ & $\begin{array}{c}\text { Receiv- } \\
\text { Der } \\
\text { Dispo- } \\
\text { sition }\end{array}$ \\
\hline & & &
\end{tabular}

(1)

\begin{tabular}{l|l}
$\mathrm{N} / \mathrm{A}$ & 2 \\
\hline
\end{tabular} 


\section{ENGINEERING DATA TRANSMITTAL}

(CONTINUATION PAGE)

5. Proj.JProg/Dept.Div.:

HANDI 2000
DAWN E. ADAMS
6. Cog. Eng.

1. EDT

625358

15.

(A)
Item

Item
No.

(B) Document/Drawing No.

\begin{tabular}{l|l}
14 & HNF -2652 \\
\hline
\end{tabular}

\begin{tabular}{|l|l}
\hline & \\
\hline & \\
\hline 15 & HNVF -2653 \\
\hline
\end{tabular}

\begin{tabular}{|l|l|}
\hline & \\
\hline &
\end{tabular}

\begin{tabular}{|l|l|}
\hline 16 & HNF -2654 \\
\hline
\end{tabular}

要

17 HNF-2655

\begin{tabular}{|l|l}
\hline 18 & HNF -2656 \\
\hline
\end{tabular}

$19 \quad$ HNF -2658

$+1$

20 HNF -2659

\begin{tabular}{|l|l|}
\hline & \\
\hline & \\
\hline & \\
\hline
\end{tabular}

\begin{tabular}{|l|l}
\hline 21 & HNF -2661 \\
\hline
\end{tabular}

\begin{tabular}{|}
\hline \\
\hline
\end{tabular}

22

HNF -2662

(CONTINUED ON PG 4)
(F)

DATA TRANSMITTED

\begin{tabular}{c|c|}
\hline (C) & (D) \\
Sheet & Rev. \\
No. & No.
\end{tabular}
(E) Title or Description of Data Transmitted

\section{(F)}

Approval nator

(G)

(G)

\begin{tabular}{|l|l|}
\hline N/A & 0 \\
\hline & \\
\hline
\end{tabular}

\begin{tabular}{|l|l|l|} 
& & \\
N/A & 0 & \\
\hline & &
\end{tabular}

UNIT BILLED SERVICES FOR

HANDI 2000 BUSINESS

MANAGEMENT SYSTEM

OTS CASH DISBURSEMENT

PROCESS FOR BUSINESS

MANAGEMENT SYSTEM

TRAVEL EXPENSE

REIMBURSEMENT FOR HANDI

2000 BUSINESS MANAGEMENT

SYSTEM

CASH RECEIPTS PROCESS FOR

HANDI 2000 BUSINESS

MANAGEMENT SYSTEM

INSURANCE PAYMENT PRBCESS N/A 2

FOR HANDI 2000 BUSINESS

MANAGEMENT SYSTEM

SAVINGS PLAN PAYMENT

PROCESS FOR HANDI 2000

BUSINESS MANAGEMENT

SYSTEM

\begin{tabular}{|l|l|l} 
WORK PERFORMED BY ENTER- & N/A & 2 \\
\hline
\end{tabular}

PRISE COMPANIES (ENCO)

FOR (PHMC) FOR HANDI 2000

BUSINESS MANAGEMENT

SYSTEMS

WORK PERFORMED BY PHMC

FOR OHC-WORK ORDER FOR

HANDI 2000 BUSINESS

MANAGEMENT SYSTEM

WORK PERFORMED BY OHC FOR N/A 2

PHMC-WORK ORDER PROCESS

FOR HANDI 2000 BUSINESS

MANAGEMENT SYSTEM
Page 3 of 4

(H)

\begin{tabular}{|l|l|}
\hline (H) & (l) \\
\hline
\end{tabular}

Reason
for
Trans-
mittal

Origi-

nator

Receiv-

mittal

stion

Dispo-
sition 
ENGINEERING DATA TRANSMITTAL

(CONTINUATION PAGE)

5. Proj./Prog./Dept.Div.:

HANDI 2000

\author{
6. Cog. Eng. \\ DAWN E. ADAMS
}

DATA TRANSMITTED

15.

23 HNF -2663

\begin{tabular}{|c|c|c|c|}
\hline ing No. & $\begin{array}{l}\text { (C) } \\
\text { Sheet } \\
\text { No. }\end{array}$ & $\begin{array}{l}\text { (D) } \\
\text { Rev. } \\
\text { No. }\end{array}$ & $\begin{array}{l}\text { (E) Title or Description of Data } \\
\text { Transmitted }\end{array}$ \\
\hline & $\mathrm{N} / \mathrm{A}$ & 0 & WALK-IN WORK FOR OTHER \\
\hline & & & HANEORD CONTRACTORS FOR \\
\hline & & & HANDI 2000 BUSINESS \\
\hline & & & MANAGEMENT SYSTEM \\
\hline & $N / A$ & 0 & DOE HANFORD REQUEST FOR \\
\hline & & & SERVICES FOR HANDI 2000 \\
\hline & & & BUSINESS MANAGEMENT \\
\hline & & & SYSTEM \\
\hline
\end{tabular}

\begin{tabular}{|c|c|c|c|}
\hline (F) & $(\mathrm{G})$ & $(\mathrm{H})$ & $(\mathrm{I})$ \\
\hline $\begin{array}{c}\text { Approval } \\
\text { Desig- } \\
\text { nator }\end{array}$ & $\begin{array}{c}\text { Reason } \\
\text { for } \\
\text { Trans- } \\
\text { mittal }\end{array}$ & $\begin{array}{c}\text { Origi- } \\
\text { nator } \\
\text { Dispo- } \\
\text { stion }\end{array}$ & $\begin{array}{c}\text { Receiv- } \\
\text { er } \\
\text { Dispo- } \\
\text { sition }\end{array}$ \\
\hline N/A & 2 & & \\
\hline
\end{tabular}




\title{
DOE HANFORD REQUEST FOR SERVICES FOR HANDI 2000 BUSINESS MANAGEMENT SYSTEM
}

\author{
Diane Wilson, Fluor Daniel Hanford Co. \\ MSN G1-22, 2355 stevens \\ Richland, WA 99352 \\ U.S. Department of Energy Contract DE-AC06-96RL13200 \\ $\begin{array}{ll}\text { EDT/ECN: } & 625358 \\ \text { Org Code: } & \text { FK260000 } \\ \text { B\&R Code: } & \text { EW70070100 }\end{array}$ \\ UC: 900 \\ Charge Code:YBPME \\ Total Pages:

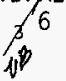

Key Words: RES process,

Abstract: This woxk includes services provided by PHMC which are defined by DOE-RL with support from FDH Accounting. Services include:--Site wide information system support, Site infrastructure including occupancy, Utilities, Fleet services, printing and reproduction services, Training, supplies and material, etc.

TRADEMARK DISCLAIMER. Reference herein to any specific commercial product, process, or service by trade name, trademark, manufacturer, or otherwise, does not necessarily constitute or imply its endorsement, recommendation, or favoring by the United States Government or any agency thereof or its contractors or subcontractors.

Printed in the United States of America. To obtain copies of this document, contact: Document Control Services, P.O. Box 950, Mailstop H6-08, Richland WA 99352, Phone (509) 372-2420; Fax (509) 376-4989.

PeopleSoft is a trademark of Indus Corp. Passport is a trademark of Indus Corp.

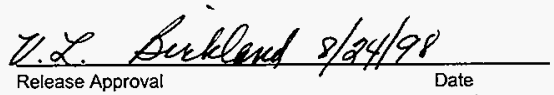

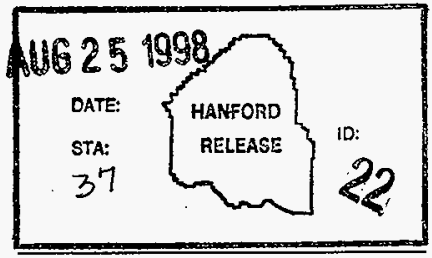

Release Stamp

\section{Approved For Public Release}




\section{Memorandum}

To:
D.E. Adams G1-21
M.J. Byrd
$\mathrm{H} 2-08$,
A.A. Carter
$\mathrm{H} 2-19$,
C.R. Hopkins
G1-57,

K.B. Adamsons H2-19

R.C. Corless H2-19,

J.A. Caldwell G1-22,

R.J. McFadzean H2-19

From: P. C. Felts

Date: April 17, 1998

Re: PeopleSoft General Ledger / Project Costing - Business System Implementation Sessions Final Documentation

\section{HANDI 2000 - Finance}

Attached for your information is the complete set of documentation of the work flow analysis from the Business System Implementation (BSI) Sessions.

One of the goals of finalizing documentation from the BSI Sessions is to use the documents as a communication tool.

Other goals of the BSI sessions were to validate the As-Is process, and describe the vision of the To-Be process that fit the baseline software processes. In the future, this documentation will be accessible electronically via a read-only shared area.

I thank you and your staff for your support during this information gathering and envisioning stage of the new system implementation project

If you have any questions, I can be reached on 376-0367 or via email.

Attachment 
Date:

$4 / 17 / 98$

Documentation: Finance Business System Implementation (BSI) Sessions

Processes Included:

- Accrual

- Allocations

- Budget

- Fit-Gap Analysis

- Closing Procedure

- Fixed Assets Accounting

- Funds Control

- General Ledger Account Maintenance

- Hanford Business Structure

- Inventory Processes

- Invoice/DISCAS

- Labor Costing

- Passport-PeopleSoft Integration

- Unit Billed Service

- Travel \& Treasury:

- OTS Cash Disbursement Process

- Travel Expense Reimbursement

- Cash Receipt Process

_ Savings, Pensions, \& Insurance:

- _ Insurance Payment Process

- Pension Payment Process

- Savings Plan Payment Process

- Other Hanford Contractors:

- Worked Performed by Enco's for PHMC

- Worked Performed by Enco's for OHC

- Worked Performed by PHMC for OHC - Work Order

- Worked Performed by OHC for PHMC - Work Order Process

- Worked Performed by PHMC for OHC - Walk In

- Worked Performed by PHMC for DOE-Richland using the Request for Service Process

FDH Project Lead: Name

Signature

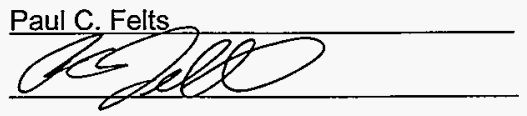




\section{Work Performed by PHMC for DOE-Richland using the Request For Service (RFS) process}

\section{Overview}

This work includes services provided by PHMC which are defined by DOE-RL with support from FDH Accounting. Services include:

- Site wide information system support

- Site infrastructure including occupancy

- Utilities

- Fleet services

- Printing and reproduction services

- Training

- Supplies and material, etc.

\section{Assumptions}

$\square$ The as-is process will not change. This assumption is mission critical.

\section{Policies}

All PHMC costs will be accumulated and controlled in PeopleSoft Projects module.

\section{As-Is Procedures}

1. Plan for work is set up

FDH Contracts and FDH Accounting receives RFS including funding from DOE and sets up WBS structure as required.

\section{PHMC performs work}

PHMC performs the work as per RFS and accumulates costs.

3. FDH invoices DOE-RL

FDH invoices DOE-RL for cost against appropriate funding source (general ledger account 8132).

\section{To-Be Procedures}

There is no change planned for this process except for system replacement. The work will be set up in PeopleSoft Projects.

Impacts

- None

Issues \& Action Items

Issues

D None

Action Items 
- Confirm as-is process is not changing. Party responsible: Dick Pouley/Roger Corless Due Date: May 1 (This action does not delay prototyping). 
Other Hanford Contractors (Work For Others) Process - Work Performed by PHMC for DOERL using Request for Service (RFS) process.

"As Is"

"To Be"

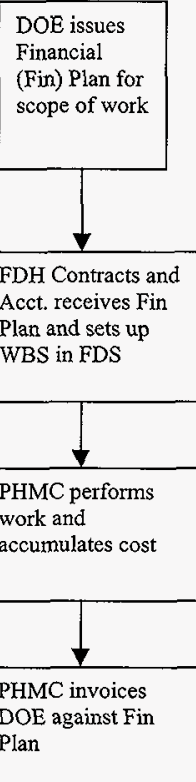

